*Correspondencia:

tamaradelgado@live.com

Departamento de Anatomía patológica. Instituto Oncológico Nacional, Avda. Pedro Menéndez Gilbert, junto a la Cdla Atarazana, Guayaquil CP 090505.

Conflicto de intereses: LoS autores declaran no tener conflictos de intereses.

Recibido: 8 de Febrero 2021

Aceptado: 21 de Marzo, 2021

Publicado: 30 de Abril, 2021

Editor: Dr. Felipe Campoverde

Membrete bibliográfico:

Delgado T, Tortoledo M. Impacto en la reducción de la morbilidad utilizando la técnica de ganglio centinela en pacientes con cáncer de mama. Rev. Oncol. Ecu 2021;31(1):66-73.

ISSN: 2661-6653

DOI: https://doi.org/10.33821/549

Copyright Delgado T, et al. Este artículo es distribuido bajo los términos de Creative Commons Attribution License, el cual permite el uso y redistribución citando la fuente y al autor original.

\section{Impacto en la reducción de la morbilidad utilizando la técnica de ganglio centinela en pacientes con cáncer de mama.}

\section{Impact on the reduction of morbidity using the senti- nel node technique in patients with breast cancer}

\section{Tamara Delgado Dávila*1 (D), María Tortoledo Rodríguez1}

1. Servicio de Anatomía Patológica, SOLCA Guayaquil.

\section{Resumen}

Introducción: El cáncer de mama afecta cada vez más a las mujeres a nivel mundial. Los tipos de tratamiento quirúrgico y quimioterápico han evolucionado, consecuentemente es necesaria la correcta evaluación del tumor primario y los ganglios linfáticos implicados por ser un importante factor pronóstico y tratamiento. La técnica de ganglio centinela evalúa al primer ganglio en recibir el drenaje linfático del tumor.

Métodos: el objetivo fue determinar el impacto en pacientes con cáncer de mama en un estudio cuantitativo, observacional no experimental, correlacional de recolección retrospectiva, de tipo cohorte histórico. Se recuperó 153 pacientes en dos grupos de estudio, los que se sometieron a la técnica de ganglio centinela y los que se realizaron linfadenectomía axilar.

Resultados: el tipo histológico de cáncer de mama más frecuente fue el ductal infiltrante de grado histológico 2 , un $76.4 \%$ y $73.6 \%$ de pacientes tuvieron receptores de estrógeno y progesterona positivo respectivamente mientras el receptor 2 de factor de crecimiento epidérmico humano fue positivo en 16.9\%. La mediana del número de ganglios metastásicos fue semejante en los dos grupos, no así el número de ganglios libres 3 vs. 14 respectivamente $(P<0.001)$. En el estudio se evidenció morbilidad en $23.1 \%$ de pacientes que se biopsiaron el Ganglio Centinela, en contraste con $45.5 \%$ de en quienes no se efectuó dicho procedimiento $(P=0.025)$, la morbilidad más frecuentemente asociada fue el edema de la extremidad (27\%).

Conclusiones: se demostró que la linfadenectomía expone tres veces a desarrollar morbilidades en comparación con la técnica de ganglio centinela.

Palabras claves:

DeCS: Biopsia del Ganglio Linfático Centinela, Ganglio Linfático Centinela, Neoplasias de la Mama, morbilidad, Escisión del Ganglio Linfático.

DOI: $10.33821 / 549$ 


\begin{abstract}
Introduction: Breast cancer affects more and more women worldwide. The types of surgical and chemotherapeutic treatment have evolved, consequently the correct evaluation of the primary tumor and the involved lymph nodes is necessary because it is an important prognostic factor and treatment. The sentinel lymph node technique evaluates the first node in receiving the lymphatic drainage of the tumor.
\end{abstract}

Methods: the objective was to determine its impact in patients with breast cancer in a quantitative, observational, non-experimental, correlational, retrospective, historical cohort study. We recovered 153 patients in two study groups, those who underwent the sentinel lymph node technique and those who underwent axillary lymphadenectomy.

Results: the most frequent histological type of breast cancer was the infiltrative ductal of histological grade $2,76.4 \%$ and $73.6 \%$ of patients had positive estrogen and progesterone receptors respectively while the receptor 2 of human epidermal growth factor was positive in $16.9 \%$. The median number of metastatic lymph nodes was similar in the two groups, but not the number of free nodes 3. 14 respectively $(P<0.001)$. In the study, morbidity was evidenced in $23.1 \%$ of patients who were sentinel lymph node biopsies, in contrast to $45.5 \%$ of those who did not undergo the procedure $(P=0.025)$, the most frequently associated morbidity was the edema of the extremity (27\%).

Conclusions: lymphadenectomy was shown three times to develop morbidities compared to the sentinel lymph node technique.

\title{
Keywords:
}

MESH: Sentinel Lymph Node Biopsy; Sentinel Lymph Node; Breast Neoplasms; Morbidity; Lymph Node Excision.

DOI: $10.33821 / 549$

\section{Introducción}

El cáncer de mama es un tumor con una ocurrencia creciente. Últimamente la supervivencia ha aumentado debido a la detección precoz y tratamiento mejorado como quimioterapia con blancos moleculares, radioterapia y cirugía dependiendo de su estadío, características del tumor y ganglios linfáticos implicados. El procedimiento quirúrgico tradicionalmente sinónimo de mastectomía radical ha sido modificado por nuevos protocolos quirúrgicos que tienen por objeto mejorar la calidad de vida [1].

En el cáncer de mama el estado histológico de los ganglios linfáticos axilares es un importante factor pronóstico y su evaluación se traducirá en una mejora en el tratamiento y consecuentemente el bienestar del paciente. El vaciamiento axilar ha sido el procedimiento habitual para evidenciar la presencia de metástasis ganglionares no obstante, luego de realizado el estudio anatomopatológico un alto porcentaje de ganglios se reportaban negativos [2].

La técnica biopsia selectiva de ganglio centinela (BSGC) ha sido catalogada como una nueva conducta terapéutica eficaz al evaluar ganglios linfáticos con el fin de evitar una linfadenectomía innecesaria en aquellas pacientes con cáncer de mama en las que sus ganglios están 
libres de tumor. Para localizarlo durante la cirugía se realiza el marcaje linfático previo con un procedimiento que utiliza un coloide más un radiotrazador como el Tecnesio (Tc99); una vez ubicado se envía al departamento de Patología en donde mediante sección congelada se analiza y reporta el resultado, de ser negativo evitará a la paciente la resección axilar [3].

Mediante la BSGC se puede predecir el estado de los demás ganglios linfáticos basado en la teoría que existe un drenaje ordenado y que un primer ganglio sirve de filtro hacia los demás. Veronesi, y colaboradores, (1997) [4] en un estudio que sentó precedentes indicaron que el GC debe ser bisecado a lo largo de su eje e incluído para estudio intraoperatorio por congelación. Se envían un máximo de tres ganglios y todos serían examinados en su totalidad, obteniéndose varios niveles teñidos con Hematoxilina \& Eosina.

Este procedimiento se ha venido realizando desde hace varios años en centros hospitalarios fuera del país por lo tanto el propósito de esta investigación fue fundamentar evidencia obtenida del Instituto Oncológico Nacional SOLCA con el objeto de demostrar que con la evaluación del ganglio centinela se puede evitar complicaciones prevenibles para el beneficio de las pacientes.

\section{Materiales y métodos \\ Diseño del estudio}

El presente estudio es observacional, analítico, con un grupo de comparación histórico.

\section{Área de estudio}

El estudio se realizó en el servicio de anatomía patológica del Instituto Oncológico Nacional "Dr. Juan Tanca Marengo" de la Sociedad de Lucha contra el Cáncer-SOLCA, Guayaquil Ecuador, el período estudio fue del 1ro de enero del 2018 al 31 de diciembre del 2019.

\section{Universo y muestra}

El universo fue conformado por todos los pacientes hospitalizados en la institución en quienes se realizó una biopsia del ganglio centinela y con cáncer de mama. El cálculo del tamaño muestral fue no probabilístico, tipo censo en donde se incluyeron todos los casos posibles de la institución en las fechas establecidas.

\section{Participantes}

Se incluyeron casos en quienes se registraron el ingreso por primera ocasión a la institución con la necesidad de biopsia del ganglio centinela por cáncer de mama. El grupo de estudio fue conformado por el grupo en quienes se realizó la técnica de BSGC, el grupo control lo constituyeron pacientes en quienes se realizó disección axilar ganglionar. Se excluyeron casos en quienes no se completó las variables estudiadas. 


\section{Variables}

Las variables fueron de tipo demográficas como edad, sexo. Características del tumor como tamaño, grado, tipo, tipo, perfil inmunohistoquímico, afectación ganglionar, biopsia de ganglio centinela, ganglios con metástasis, ganglios libres. Se recolectaron variables clínicas de morbilidad.

\section{Procedimientos, técnicas e instrumentos.}

Los datos fueron recogidos de la historia clínica en un formulario diseñado exclusivamente para tal propósito. Adicionalmente se consultaron registros de anatomía patológica, formularios de flebología para obtener los datos secundarios y complementarios al estudio. Para garantizar la confiabilidad de la información los investigadores fueron entrenados sobre la recolección de los datos.

\section{Análisis estadístico}

Recopilada la información se ingresó en una matriz de datos del software SPSS ${ }^{\text {TM }} 22.0$ (IBM, Armonk, NY, EEUU). Se utilizó estadística descriptiva en base a frecuencias y porcentajes para las variables cualitativas y para las cuantitativas medidas de tendencia central. Ganglio Centinela. Se empleó la prueba T de Student o U de Mann-Whitney para el contraste entre variables continuas, y chi-cuadrado de Pearson o de Fisher para el contraste entre variables categóricas, según corresponda. Se estableció la asociación entre la no realización de Ganglio Centinela vs. la presencia de morbilidades entre aquellos pacientes quienes no presentaron linfonodos asociados, mediante riesgo relativo (RR) y número necesario a tratar (NNT). Un valor $P<0.05$ fue considerado como estadísticamente significativo.

\section{Resultados}

El estudio incluyó 153 pacientes.

\section{Caracterización demográfica}

Se recuperó un total de 153 pacientes en dos grupos los que se realizaron la técnica de ganglio centinela 52/153 y los que se sometieron a disección ganglionar 101/153. La edad promedio de mujeres con cáncer de mama fue de 55.8 años, el tamaño tumoral 2.4cm (pT2). El tipo histológico de cáncer de mama más frecuente fue el ductal infiltrante presente en 116/153 (75.8\%) pacientes, así como el grado 2 en la escala de Nottingham en 84/153 (59.6\%) pacientes; No hubo diferencia estadísticamente significativa entre dichas variables y los grupos de estudio. los receptores de estrógeno y progesterona positivo se presentaron en 113/153 (76.4\%) y 109/153 (73.6\%) respectivamente. El HER2 fue positivo en 25/153 (16.9\%) pacientes, de los cuales 5/25 correspondieron al grupo en que se practicó técnica Ganglio Centinela $(P=0.011)$. Ki 67 presentó una mediana de 25\% (2 - 95\%), y la invasión linfovascular fue positiva en 20/153 pacientes. A excepción de HER2, no hubo diferencia estadísticamente significativa entre dichas variables y los grupos de estudio.

En quienes se practicó la técnica de Ganglio Centinela 19/52 (36.5\%) fueron positivos para malignidad. En este grupo se resecó una mediana de 4 ganglios en comparación con 16 en el grupo en quienes no se realizó dicha técnica $(P<0.001)$. De éstos la mediana del número de 
ganglios metastásicos fue semejante, no así el número de ganglios libres: 3 (1 -31) vs. 14 (3 - 33) respectivamente $(P<0.001)$. Tabla 1.

La BSGC redujo en un 84\% la presencia de morbilidades asociadas a un vaciamiento ganglionar no necesario (RR 3.07; IC 95\% 1.32 - 7.14; $P<0.001$ ) (Vea la tabla 2). El número de pacientes necesario a tratar (NNT) a fin de evitar un caso de morbilidad fue de 3.192 es decir que es preciso realizarle técnica de Ganglio Centinela a tres pacientes para prevenir en un caso el ulterior desarrollo de morbilidades asociadas a una linfadenectomía no necesaria (tabla $\underline{3})$.

Tabla 1 Resultado de la técnica de ganglio centinela en la población general y según cada grupo de estudio.

\begin{tabular}{|c|c|c|c|c|c|c|c|c|}
\hline \multirow{3}{*}{$\begin{array}{c}\text { Ganglio centinela }(\mathrm{n}= \\
52), \mathrm{n}(\%)\end{array}$} & \multirow{3}{*}{$\begin{array}{l}\text { Positivo } \\
\text { Negativo }\end{array}$} & \multicolumn{2}{|c|}{$\begin{array}{l}\text { General } \\
(n=153)\end{array}$} & \multicolumn{2}{|c|}{$\begin{array}{l}\text { BSGC } \\
(n=52)\end{array}$} & \multicolumn{2}{|c|}{$\begin{array}{l}\text { No realización de } \\
\text { Ganglio Centinela } \\
\quad(n=101)\end{array}$} & \multirow{3}{*}{$\begin{array}{c}\mathrm{P} \\
--\end{array}$} \\
\hline & & 19 & $(36,5)$ & 19 & $(36,5)$ & 0 & & \\
\hline & & 33 & $(63,5)$ & 33 & $(63,5)$ & 0 & & \\
\hline \multicolumn{2}{|c|}{ Ganglios centinela, mediana (rango) } & 1.0 & $(1-4)$ & 1.0 & $(1-4)$ & 3.0 & & 0.057 \\
\hline \multicolumn{2}{|c|}{ Ganglios satélite, mediana (rango) } & 1.0 & $(0-5)$ & 1.0 & $(0-5)$ & 5.0 & & 0.085 \\
\hline \multicolumn{2}{|c|}{ Ganglios resecados, mediana (rango) } & 13.0 & $(1-34)$ & 4.0 & $(1-32)$ & 16.0 & $(4-34)$ & $<0.01$ \\
\hline \multicolumn{2}{|c|}{ Ganglios metastásicos, mediana (rango) } & 0.0 & $(0-18)$ & 0.0 & $(0-18)$ & 0.0 & $(0-17)$ & 0.805 \\
\hline \multirow{2}{*}{\multicolumn{2}{|c|}{$\begin{array}{l}\text { Ganglios libres, mediana (rango) } \\
\text { Tamaño de la metástasis (cm), mediana } \\
\text { (rango) }\end{array}$}} & 11.0 & $(1-33)$ & 3.0 & $(1-31)$ & 14.0 & $(3-33)$ & $<0.01$ \\
\hline & & 1.0 & $(1-6)$ & 1.0 & $(1-6)$ & 1.0 & & 0.451 \\
\hline
\end{tabular}

BSGC: biopsia selectiva del ganglio centinela

Tabla 2 Complicaciones posteriores a la realización de linfadenectomía, en la población general y según cada grupo de estudio

\begin{tabular}{|c|c|c|c|c|c|c|c|}
\hline & \multicolumn{2}{|c|}{$\begin{array}{l}\text { General } \\
(n=153)\end{array}$} & \multicolumn{2}{|c|}{$\begin{array}{l}\text { BSGC } \\
(n=52)\end{array}$} & \multicolumn{2}{|c|}{$\begin{array}{c}\text { No realización de } \\
\text { Ganglio Centinela } \\
(n=101)\end{array}$} & \multirow{2}{*}{$\begin{array}{c}\text { valor } \\
p\end{array}$} \\
\hline Morbilidad $(\mathrm{n}=141), \mathrm{n}(\%)$ & & & & & & & \\
\hline Sí & 58 & $(41.1)$ & 12 & $(23.1)$ & 46 & $(45.5)$ & \\
\hline Edema & 19 & (13.5) & 3 & $(6.4)$ & 16 & $(17.0)$ & \\
\hline Parestesia & 1 & $(0.7)$ & 0 & & 1 & $(1.1)$ & \\
\hline $\begin{array}{l}\text { Limitación a la movili- } \\
\text { dad }\end{array}$ & 4 & (2.8) & 0 & & 4 & (4.3) & \\
\hline Celulitis & 1 & $(0.7)$ & 0 & & 1 & $(1.1)$ & \\
\hline Dolor & 10 & (7.1) & 2 & (4.3) & 8 & $(8.5)$ & \\
\hline Varios & 13 & $(9.2)$ & 2 & $(4.3)$ & 11 & $(11.7)$ & \\
\hline Otros & 10 & (7.1) & 5 & $(10.6)$ & 5 & $(5.3)$ & \\
\hline No & 83 & $(58.9)$ & 35 & $(74.5)$ & 48 & $(51.1)$ & \\
\hline
\end{tabular}

Tabla 3 Relación entre la realización de Ganglio Centinela, la presencia de linfonodos metastásicos, y el desarrollo de morbilidades ulteriores.

\begin{tabular}{|c|c|c|c|c|}
\hline & \multicolumn{2}{|c|}{$\begin{array}{l}\text { No linfonodos metastásicos } \\
\qquad(n=104)\end{array}$} & \multicolumn{2}{|c|}{$\begin{array}{c}10>\text { linfonodos metastásicos } \\
(n=49)\end{array}$} \\
\hline & Morbilidades & No morbilidades & Morbilidades & No morbilidades \\
\hline No realización de Ganglio Centinela, n (\%) & $33(86.8)$ & $38(57.6)$ & $14(66.6)$ & $16(57.1)$ \\
\hline Ganglio Centinela realizado, n (\%) & $5(13.2)$ & $28(42.4)$ & $7(33.3)$ & $12(42.9)$ \\
\hline
\end{tabular}




\section{Discusión}

La realización de mastectomía con vaciamiento ganglionar en aquellos casos en que la técnica de ganglio centinela estuviere indicada conlleva una potencial exposición a comorbilidades prevenibles [5]. El objetivo de la presente investigación fue determinar el impacto de esta técnica en términos de reducción de complicaciones asociadas a la resección linfática en patología mamaria maligna.

En la población que se realizó la técnica de ganglio centinela el 98.1\% correspondió a estadío T2 o menor, 94.3\% a estadío N2a o menor y en ningún caso metastásico (M1); tal como versa el consenso español sobre biopsia selectiva de ganglio centinela realizado por Bernet y cols, 2014, en España, [6] en quienes se realizó disección axilar el 100\% correspondió a estadio T2 o menor $(P=0.211) ; 93.1 \%$ estadío N2a o menor $(P=0.016)$ y solo en un caso metastásico (0.472). Las características socio-demográficas y clínico-patológicas fueron semejantes en ambos grupos de estudio.

Se obtuvo por lo menos un ganglio centinela positivo en 19/52 (36.5\%) de los casos, de ellos 13.5\% con invasión linfovascular; en ambos grupos de estudio la mediana de la lesión tumoral fue de $1 \mathrm{~cm}(P=0.451)$ esto es particularmente importante si se recuerda la conclusión de Dash y cols, 2013 en Reino Unido [7] en donde el 26.7\% de pacientes que se realizaron biopsia selectiva de ganglio centinela fueron positivos no obstante el $63 \%$ tuvieron invasión linfovascular, indicaron que el tamaño del tumor predice significativamente el compromiso de nódulos axilares $(P=0.046)$.

El porcentaje de casos de quienes presentaron morbilidad asociada al vaciamiento ganglionar fue del $45,5 \%(P<0.05)$. El linfedema fue observado en $17 \%$ de las pacientes que se realizaron linfadenectomía así como la limitación de la movilidad del brazo en un 4.3\% de ellas. Tales complicaciones y en porcentajes similares fueron ya mencionadas en un estudio realizado por Galimberti, y cols, 2013 en Italia [8] en el que 13\% de las pacientes que se realizaron disección axilar presentaron linfedema $(P<0.001)$ y $8 \%$ presentaron limitación en la movilidad descrita como neuropatía motora $(P=0.0004)$, concluyeron que la disección axilar puede ser obviada en pacientes con cáncer de mama en estadío temprano y con un ganglio centinela negativo o con limitado compromiso metastásico (micrometástasis) lo que elimina morbilidades asociadas sin efectos adversos en cuanto a supervivencia.

Se obtuvo una mediana de resección de 4 (1-32) ganglios linfáticos, y una mediana de 3 (1 - 31) ganglios libres de neoplasia en el grupo de estudio que se sometió a la BSGC. Por otro lado, en quienes no se realizó técnica la mediana de ganglios resecados fue de 16 (4 -34) $(P$ $<0.001)$ tanto como la mediana de ganglios libres resecados fue $14(3-33)(P<0.001)$. Giuliano, y cols, 2016 en Canadá [9] realizaron una investigación semejante en donde se disecó una mediana de 2 ganglios axilares mediante la técnica de ganglio centinela vs. 17 ganglios axilares prescindiendo del protocolo $(P<0.001)$ este estudio concluye que la realización de la técnica ganglio centinela ofrece un excelente control regional para pacientes con cáncer de mama tratadas con terapia conservadora de mama y terapia sistémica adyuvante. 


\section{Conclusiones}

En la población estudiada tanto las pacientes en quienes se practicó técnica Ganglio Centinela como en quienes no se practicó tal técnica presentaron características socio-demográficas y clínico-patológicas semejantes. La técnica Ganglio Centinela permite reducir significativamente el número de vaciamientos ganglionares innecesarios en aquellas pacientes quienes no tienen infiltración ganglionar. La técnica Ganglio Centinela presentó un impacto clínico positivo en término de prevención de desarrollo ulterior de morbilidades asociadas a una linfadenectomía no necesaria en pacientes con cáncer de mama estadio T1-T2.

\section{Agradecimientos}

Los autores agradecen a todas las personas de la Institución que colaboraron en el desarrollo de esta investigación.

Nota del Editor

La Revista Oncología Ecu

permanece neutral con respecto a los reclamos jurisdiccionales en mapas publicados y afiliaciones institucionales.

\section{Información administrativa}

\section{Abreviaturas}

BSGC: Biopsia selectiva del ganglio centinela. NNT: Número necesario a tratar.

\section{Archivos Adicionales}

Ninguno declarado por los autores.

\section{Fondos}

Los autores no recibieron ningún tipo de reconocimiento económico por este trabajo de investigación. Los gastos producidos en la investigación fueron asumidos por los investigadores.

Disponibilidad de datos y materiales

Existe la disponibilidad de datos bajo solicitud al autor de correspondencia. No se reportan otros materiales.

Contribuciones de los autores

Todos los autores realizaron por igual la conceptualización, curación de datos, análisis formal, adquisición de fondos, investigación, recursos, software, redacción - borrador original, supervisión, validación, visualización, metodología, administración de proyecto, escritura: revisión y edición. 
Todos los autores leyeron y aprobaron la versión final del manuscrito.

Aprobación del comité de ética

El protocolo de investigación fue aprobado por el Departamento de Docencia del Hospital Solca - Guayaquil.

Consentimiento para publicación

El presente estudio es un análisis de base de datos, no aplica para este tipo de estudio.

\section{Referencias}

1. Goethals A, Rose J. Mastectomy. 2020 Jul 31. In: StatPearls [Internet]. Treasure Island (FL): StatPearls Publishing; 2021 Jan-. PMID: $\underline{30855800}$.

2. Samphao S, Eremin JM, El-Sheemy M, Eremin O. Management of the axilla in women with breast cancer: current clinical practice and a new selective targeted approach. Ann Surg Oncol. 2008 May;15(5):1282-96. doi: 10.1245/s10434-008-9863-8. Epub 2008 Mar 11. PMID: 18330650.

3. Balaya V, Guani B, Bonsang-Kitzis H, Deloménie M, Ngô C, Montero Macias R, et al. Place du ganglion sentinelle dans les cancers du col utérin débutants [Sentinel lymph node biopsy in early-stage cervical cancer: current state of art]. Bull Cancer. 2020 Jun;107(6):696-706. French. doi: 10.1016/j.bulcan.2019.06.011. Epub 2019 Oct 15. PMID: $\underline{31627905 .}$.

4. Veronesi U, Paganelli G, Galimberti V, Viale G, Zurrida S, Bedoni M, Costa A, de Cicco C, Geraghty JG, Luini A, Sacchini V, Veronesi P. Sentinel-node biopsy to avoid axillary dissection in breast cancer with clinically negative lymph-nodes. Lancet. 1997 Jun 28;349(9069):1864-7. doi: 10.1016/S0140-6736(97)01004-0. PMID: 9217757.

5. Giuliano AE, Hunt KK, Ballman KV, Beitsch PD, Whitworth PW, Blumencranz PW, Leitch AM, Saha S, McCall LM, Morrow M. Axillary dissection vs no axillary dissection in women with invasive breast cancer and sentinel node metastasis: a randomized clinical trial. JAMA. 2011 Feb 9;305(6):569-75. doi: 10.1001/jama.2011.90. PMID: 21304082; PMCID: PMC5389857.

6. Bernet L, Piñero A, Vidal -Sicart S, Dueñas B, Tresserra F, Cano A, et al.. Consenso sobre la Biopsia Selectiva del Ganglio Centinela en el Cáncer de Mama.Revisión 2013 de la Sociedad Española de Senología y Patología Mamaria. Revista Española de Patología. 2014; 263. doi: 10.1016/j.patol.2013.11.001

7. Dash I, Howell M, Schwodler K, Taylor G, Goddard D.A, McIntosh J, et al. The positive sentinel lymph node:Can we predict wich patients are of real benefit with further lymph node surgery? European Journal of Surgical Oncology. 2013;39(5):468 doi:10.1016/j.ejso.2013.01.062.

8. Galimberti V, Cole B, Zurrida S, Viale G, Luini A, Veronesi P, et al. International Breast Cancer Study Group Trial 23-01 investigators. Axillary dissection versus no axillary dissection in patients with sentinel-node micrometastases (IBCSG 23-01): a phase 3 randomised controlled trial. Lancet Oncol. 2013 Apr;14(4):297-305. doi: 10.1016/S1470-2045(13)70035-4. Epub 2013 Mar 11. Erratum in: Lancet Oncol. 2013 Jun;14(7):e254. PMID: 23491275; PMCID: PMC3935346.

9. Giuliano A, Balman K, McCall L, Beitsch P, Whitworth P, Leitch M., et al. Locoregional recurrence after sentinel Lymph node dissection with or without axillary disscetion in patients with sentinel lymph node metastases. Annals of Surgery. 2016; 264: 413-420.. discussion 432-3. doi: 10.1097/SLA.0b013e3181f08f32. PMID: 20739842; PMCID: PMC5593421. 